\title{
Application of Pixel-Oriented Visualization Technique in the Centralized Monitoring of Smart Grid Dispatching and Control System
}

\author{
Yin $Z \mathrm{ZHANG}^{1}$ \& Yansheng $L A N{ }^{1}$ \& Yang BAI ${ }^{1}$ \& Ying XING ${ }^{1}$ \& Qiang $\mathrm{LI}^{1}$ \& Jie \\ $X U^{1}$ \& Sen $\mathrm{LI}^{1}$ \&Qingshan $\mathrm{ZHU}{ }^{2}$ \\ ${ }^{1}$ China Electric Power Research Institute, Beijing, China \\ ${ }^{2}$ State Grid Zhejiang Electric Power Company, Hangzhou, China
}

\begin{abstract}
KEYWORD: smart grid; dispatching and control system; centralized maintenance; centralized monitoring; pixel-oriented visualization; big data visualization

ABSTRACT: With the rapid development of smart grid dispatching and control system in State Grid Corporation of China (SGCC), the complexity of system operation and maintenance increases sharply. In order to improve the maintenance efficiency, SGCC put forward the remote centralized maintenance mode. The centralized monitoring is the essential business of centralized maintenance, it collect the vast amounts of information from nationwide smart grid dispatching and control systems, and provides monitoring pictures for the users who need understand the global running state of the systems. How visualizing the huge information in the limit screens is a problems to be solved. This paper introduces the application of pixel-oriented visualization techniques in the centralized monitoring, takes the nationwide state estimation rate monitoring for an example, and compare with original techniques. Through the research and application, we find that using pixel-oriented visualization techniques could make centralized monitoring more effective.
\end{abstract}

\section{GENERAL INTRODUCTIONS}

\section{Centralized maintenance of smart grid dispatching and control system}

With the rapid development of smart grid dispatching and control system in State Grid Corporation of China (SGCC), the complexity of system operation and maintenance increases sharply. In order to improve the maintenance efficiency, SGCC put forward the remote centralized maintenance mode (Shuai et al. 2013, Chai et al. 2014). The remote centralized maintenance includes centralized monitoring (Zhu et al. 2009), centralized fault warning and treatment, and centralized statistical analyzing. The centralized monitoring is the essential business, it collect the running status information of nationwide smart grid dispatching and control systems into one maintenance center, and it provides monitoring pictures for the users who need understand the global running state of the systems. The information contains hardware status, operating system resources, database status, platform server status, and advanced application status. The information covers the whole dispatching and control systems of the SGCC and is real-time collected. It has big amount of data, but the number of monitor screens is only dozens. In order to make the user detect system problems comprehensively and timely, the information visualization techniques should be applied to better display the huge information in the limit screens.

\section{Visualizing vast amounts of information}

Information visualization techniques are becoming increasingly important for the analysis and exploration of big data. A major advantage of visualization techniques over other automatic data exploration and analysis techniques (from statistics, machine learning, artificial intelligence, etc.) is that visualizations allow a direct interaction with the user and provide an immediate feedback, as well as user steering, which is difficult to achieve in most nonvisual approaches. The practical importance of visual data mining techniques is therefore steadily increasing and basically all commercial data mining systems try to incorporate visualization techniques of one kind or the other. In the visualization field, a considerable number of advanced visualization techniques for multidimensional data have been proposed. The approaches include geometric projection techniques such as parallel coordinates (Inselberg 1985, Inselberg \& Dimsdale 1990), icon-based techniques (Pickett \& Grinstein 1988, Beddow 1990), hierarchical techniques (Robertson et al. 1991), graph-based techniques (Becker et al. 1995), pixel-oriented techniques (Keim 2000), and combinations theory (Ahlberg \& Shneiderman 
1994). In general, the visualization techniques are used in conjunction with some interaction techniques (Ahlberg et al. 1992, Anupam et al. 1995). This article discuss the pixel-oriented technique and its basic application in centralized monitoring, and discusses their potential impact on future work in the area of centralized monitoring of power system.

\section{Pixel-oriented visualization technique}

The basic idea of pixel-oriented techniques is to map each data value to a colored pixel and present the data values belonging to one dimension (attribute) in a separate sub window (Fig. 1). Since, in general, our techniques use only one pixel per data value, the techniques allow us to visualize the largest amount of data which is possible on current displays (up to 2,073,600 data values with 1920x1080 pixel resolution). All pixel-oriented techniques partition the screen into multiple sub windows. For data sets with $\mathrm{m}$ dimensions (attributes), the screen is partitioned into $\mathrm{m}$ sub windows one for each of the dimensions. In the case of a special class of pixel oriented techniques the querydependent techniques an additional window is provided for the overall distance. Inside the windows, the data values are arranged according to the given overall sorting, which may be data driven for the query-independent techniques or query driven for the query-dependent techniques. Correlations, functional dependencies, and other interesting relationships between dimensions may be detected by relating corresponding regions in the multiple windows.

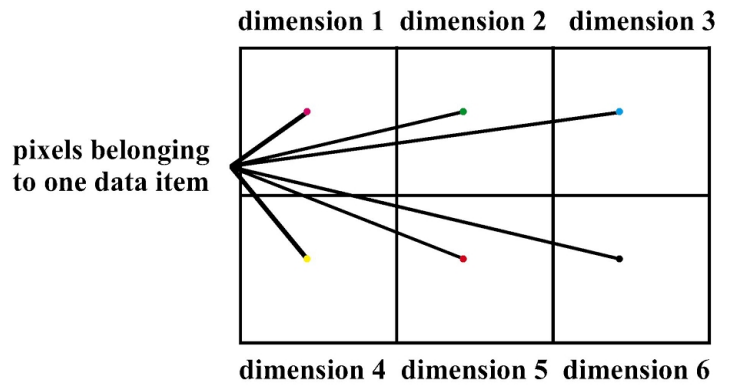

Figure 1. Basic arrangement of sub windows for data with six dimensions.

To achieve that objective a number of design problems have to be solved. The first problem is the mapping of data values to colors. A good mapping is obviously very important, but has to be carefully engineered to be intuitive. A second important question is how the pixels are arranged inside the sub windows. The arrangement depends on the data and the task of the visualization and, therefore, different arrangements are useful for different purposes. The arrangement problem can be described formally as an optimization problem and different visualization techniques optimize different variants of the optimization problem. A third question is the shape of the sub windows. With the rectangular shape of the sub windows as given in Figure 1, for data sets with a large number of dimensions, the sub windows for the different dimensions are quite distant and, therefore, it becomes difficult to find interesting relationships between the dimensions. Again, shape of sub windows can be seen as an optimization problem and we introduce a visualization technique which better solves this problem. The next question in designing the pixel-oriented techniques is how to order the sub windows for the dimensions.

\section{COLOR MAPPING}

\section{General principle}

Visualizing the data values using color corresponds to the task of mapping a single parameter distribution to color. The advantage of color over gray scales is that the number of just noticeable differences (JNDs) is much higher (Herman \& Levkowitz 1992). Finding a path through color space that maximizes the number of JNDs, but, at the same time, is intuitive for the application domain is a difficult task. For our purpose of mapping the distances to color, we can restrict the task to a simpler, more solvable problem. From a perceptual point of view, brightness is the most important characteristic for distinguishing colors corresponding to a single parameter distribution. Therefore, for our 
purpose, it is sufficient to find a color scale with a monotonically increasing (decreasing) brightness while using the full color range. The parameters of the color mapping should therefore use a monotonically decreasing brightness (intensity, lightness and value), a color ranging over the full color scale (hue), and a constant (full) saturation. We found experimentally that a color-map with colors ranging from yellow over green, blue, and red to almost black is a good choice for the color-map to be intuitive.

\section{Generating color scales}

The linear interpolation of the HSI model (H: Hue, S: Saturation, I: Intensity) scales whose lightness ranges continuously from light to dark. The hue is defined as the angle $\alpha$ between red and the chosen color.

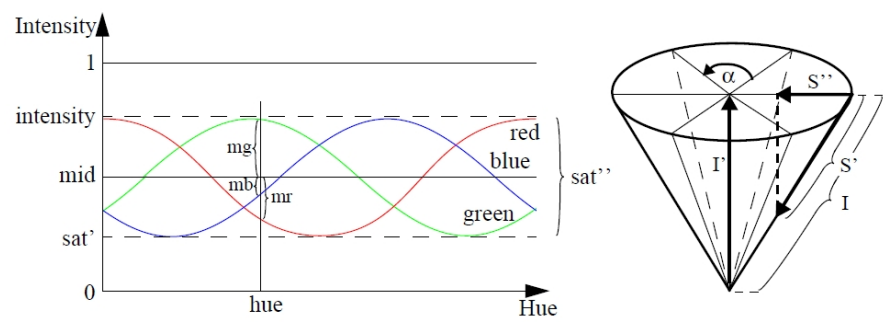

Figure 2. The HSI color model.

When moving on a circle of the HSI color cone, the red, green, and blue portion of the color follow cosine-curves with phase-shifts of $2 \pi / 3$ (Fig. 2). From the HSI color theory (Keim \& Kriegel 1995) we note that:

$$
\begin{aligned}
& \text { mid }=\frac{\text { red }+ \text { green }+ \text { blue }}{3} \\
& \text { hue }=\arccos \left(\frac{(2 \times m r-m g-m b)}{\left.\sqrt{6} \times \sqrt{m r^{2}+m g^{2}+m b^{2}}\right)}\right) \\
& \text { Intensity }=m i d+\sqrt{\frac{2}{3} \times\left(m r^{2}+m g^{2}+m b^{2}\right)} \\
& \text { saturation }=\frac{2 \times(\text { Intensity }-m i d)}{\text { Intensity }}
\end{aligned}
$$

We take the scale from 0 to 99 for an example, the hue of minimal HIS parameters is 1.5 that present light green color, and the hue of maximal HIS parameters is 1.0 that present yellow color. See Table 1.

Table 1. The parameters for generating the color scales presented in Figure 3.

\begin{tabular}{llcc}
\hline & Hue & Saturation & Intensity \\
\hline HSI(min) & 1.5 & 1.0 & 0.4 \\
HSI (max) & 1.0 & 1.0 & 1.0 \\
\hline
\end{tabular}




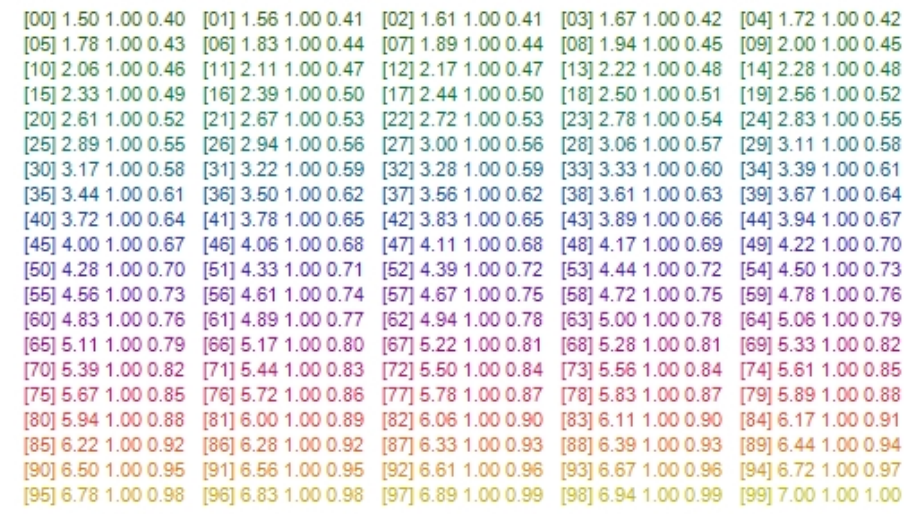

Figure 3. The example of the HSI color scale.

Figure 3.shows the results of the color scale calculation. Each point has four elements which are data value (enclosed in brackets), hue phase, saturation phase, and intensity phase. The hue phase equals the hue value divided by $\pi / 3$, the saturation phase and intensity phase has the same approach.

\section{ARRANGEMENT OF PIXELS}

The second and very important question is how the pixels are arranged within each of the sub windows. This is important since, due to the density of the pixel displays, only a good arrangement will allow a discovery of clusters and correlations among the dimensions. For the arrangement problem, we have to distinguish between data sets which have a natural ordering of data objects (such as in time-series data) and data sets, without inherent ordering (as in the case of query responses).

The pixel arrangement problem of naturally ordered data is the problem of finding a mapping of the data objects $\left\{a_{1}^{k}, K, a_{n}^{k}\right\}$ to a sub window of size $(w \times h)$, that is a bijective mapping $f$ : $\{1 \mathrm{~K} n\} \rightarrow\{1 \mathrm{~K} w\} \times\{1 \mathrm{~K} h\}$ such that

$$
\begin{aligned}
& \sum_{i=1}^{n} \sum_{j=1}^{n} \mid d(f(i), f(j)) \\
& -d\left((0,0),\left(w \cdot \sqrt{\frac{|i-j|}{n}}, h \cdot \sqrt{\frac{|i-j|}{n}}\right)\right)
\end{aligned}
$$

is minimal, where $d(f(i), f(j))$ is the $\mathrm{L}^{\mathrm{p}}$-distance of the pixels belonging to $\mathrm{a}_{\mathrm{i}}$ and $\mathrm{a}_{\mathrm{j}}$.

The definition describes the pixel arrangement problem as an optimization problem which tries to determine the arrangement of pixels which best preserves the distance of the one-dimensional ordering in the two-dimensional arrangement. The optimization formula therefore sums up over the $\mathrm{L}^{\mathrm{p}}$ distances of all pixels and normalizes them by the minimum distance of the two pixels in a rectangular sub window of proportions $(\mathrm{w} \times \mathrm{h})$. (Second part of the equation).

Even if each pixel of the display is used to represent one data value, the amount of information that can be represented using pixel-oriented techniques is still rather limited. The basic idea of query dependent visualization techniques is to visualize only the data which is relevant in the context of a specific query. Simple queries against the database can be described as regions in the k-dimensional space defined by the $\mathrm{k}$ dimensions (attributes) of the data. If exactly one query value is specified for each dimension, the query corresponds to a point in k-dimensional space; if a query range is specified for each dimension, the query corresponds to a region in k-dimensional space. The data objects which are within the query region form the result of the query. In most cases, the number of results cannot be determined a priori; the resulting data set may be quite large, or it may even be empty. In both cases, it is difficult for the user to understand the result and modify the query accordingly. To give the user more feedback on the query, we therefore do not only present the data objects which are within the query region, but also those which are ${ }^{a} \operatorname{close}^{o}$ to the query region and only approximately fulfill the query. For determining the approximate results, distances between the data and query values are calculated. The distance functions are data type and application dependent. For nu- 
meric types such as integer or real and other metric types such as date, the distance of two values is easily determined by their numerical difference. For other types such as strings, multiple distance functions such as the lexicographical difference, character-wise difference, substring difference, or even some kind of phonetic difference may be useful.

\section{SHAPE OF SUB WINDOWS}

The next important question is whether there exists an alternative to the regular partitioning of the screen into rectangular sub windows. The rectangular shape of the sub windows allows a good screen usage, but at the same time leads to a dispersal of the pixels belonging to one data object over the whole screen. Especially for data sets with many dimensions, the sub windows for the dimensions are rather far apart, which makes it difficult to detect clusters, correlations, etc. In the optimization functions described so far, the distance between the pixels belonging to one data object is not taken into account. This, however, is necessary in order to find alternative shapes for the dimension sub windows.

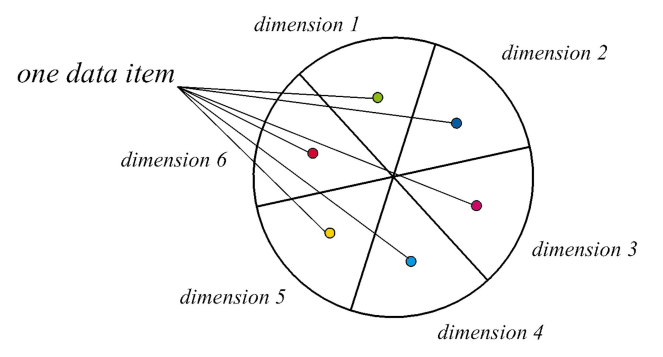

Figure 4. Alternative shape of sub windows: circle segments.

An idea for an alternative shape of the sub windows which optimizes the above function is the circle segments technique. The fundamental idea of the ${ }^{\text {a }}$ circle segments visualization technique is to display the data dimensions as segments of a circle (Fig. 4).

The mapping of the dimensions into the visual representation is fundamental for the perception of the user. The ordering of dimensions especially plays a significant role, e.g., for the detection of functional dependencies and correlations. It is therefore important to adequately arrange the dimensions. In the following, we define the dimension ordering problem mathematically as an optimization problem which ensures that the most similar dimensions are placed next to each other.

\section{Visualizing nationwide state estimation RATE}

\section{Centralized monitoring of smart grid dispatching and control system}

The centralized monitoring is the foundation of the centralized maintenance, it collect the information of nationwide smart grid dispatching and control systems such as hardware status, operating system resources, database status, platform server status, and advanced application status. The data types that already collected in centralized maintenance center is show in Figure 6. The huge information is being supervised and analyzed continuously without interruption at night and on holidays.

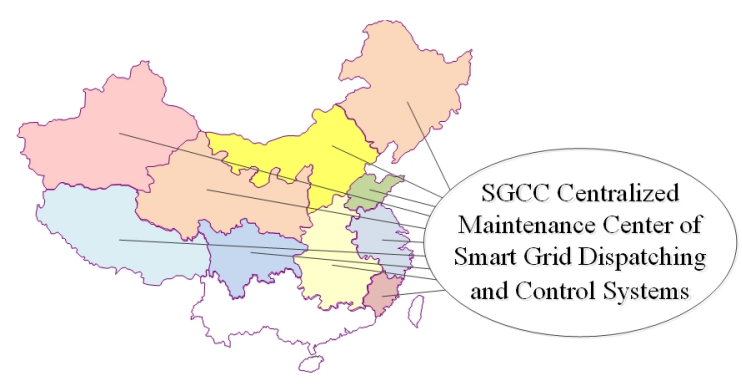

Figure 5. Centralized maintenance mode. 


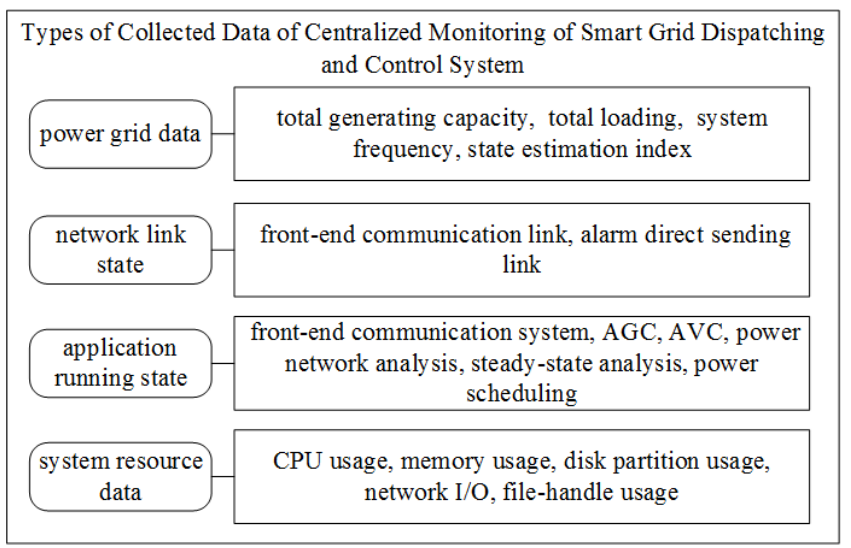

Figure 6. Types of Collected Data of Centralized Monitoring of Smart Grid Dispatching and Control System.

The centralized maintenance mode (Fig. 5) can reduce the cost of operation and maintenance of each dispatching control center, reduce the maintenance workload of staff in dispatching control center. It achieve the optimal allocation of human resources and provide powerful support and guarantee for the safe and stable operation of the Smart Grid Dispatching and Control System.

\section{Centralized monitoring of state estimation rate}

State estimation fit measurements made on the system to a mathematical model in order to provide a reliable data base for other monitoring, security assessment and control functions (Schweppe \& Handschin 1974). Its qualified rate (Fig. 7) is an important part of the dispatching and control system.

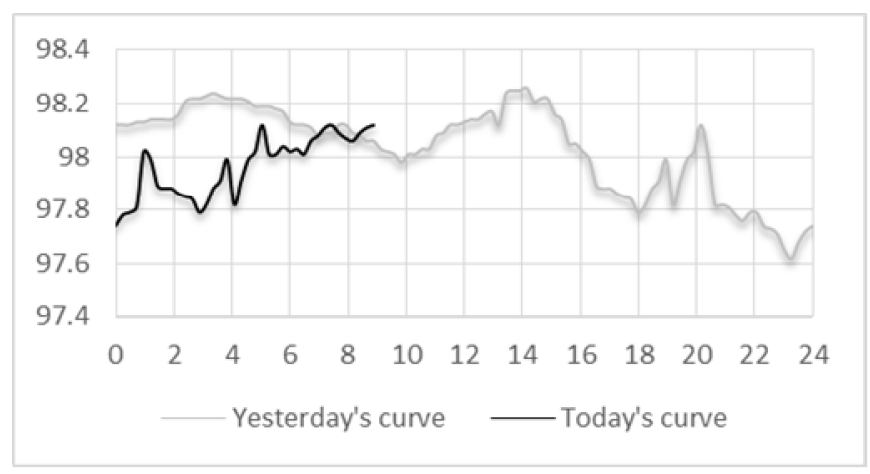

Figure 7. Classical monitoring curves of power system state estimation rate in one area.

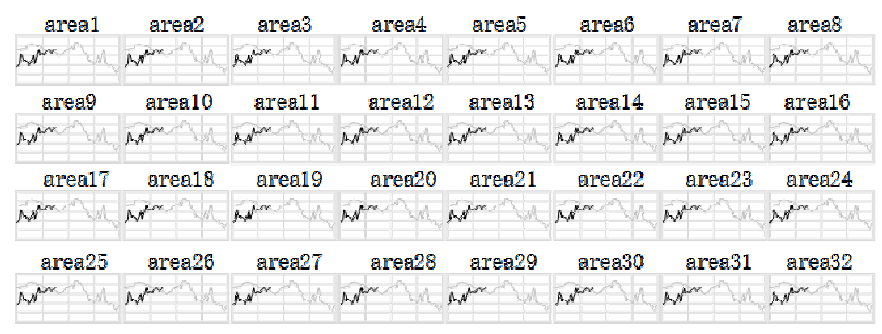

Figure 8. Monitoring 32 curves of state estimation in one screen

The number of areas being monitored in centralized maintenance center is more than one hundred. In this article we take 32 area for example. The shape of sub windows and the arrangement of pixels in monitoring state estimation is shown in Figure 9. The state estimation is calculated once per minute, so it produce 1440 values a day. The sub windows has $12 \times 240$ pixels which contains two day's information. When the number of areas raised, the classical curves (Fig. 8) become difficult to distinguish, but the pixel visualization (Fig. 10) has good visual effect. The brighter the pixel color is, 
the higher the state estimation rate is. If the rate is low to the certain extent, the pixel color will become red, and we can notice it obviously. From the pixel view we can find the relationship between each area. It is conducive to a comprehensive grasp of the overall state of the dispatching and control system.

\begin{tabular}{|c|c|}
\hline பூூூூ & பூூூூ \\
\hline பூ几ூ几レ & பூூூூ几 \\
\hline பூூூூ & பூூூூே \\
\hline பூூூூூ & பூப \\
\hline
\end{tabular}

Figure 9. The shape of sub windows and the arrangement of pixels in monitoring state estimation.
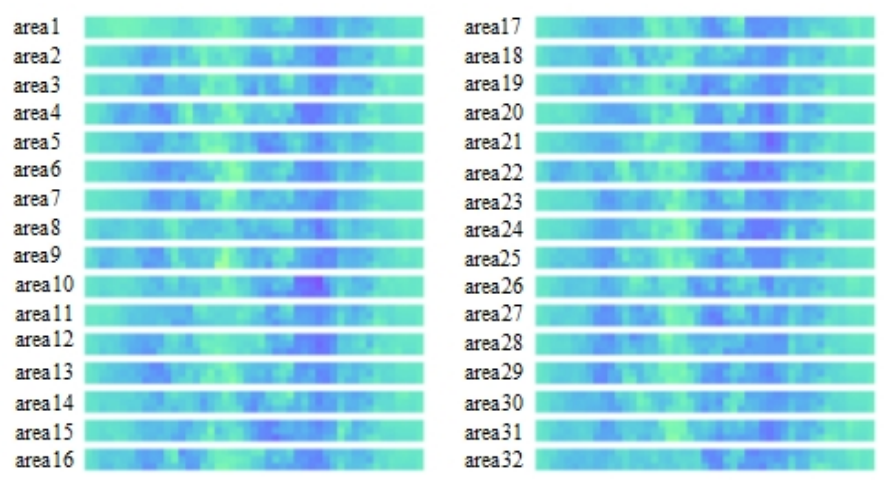

Figure 10. Monitoring state estimation in one screen with pixel visualization technique.

\section{CONCLUSIONS}

Pixel-oriented visualization techniques have been shown to be useful for the monitoring and analysis of big data. In this paper, we discuss the basic of pixel-oriented techniques, and show that underlying the development and design of pixel-oriented techniques there are a number of problems which have to be solved. Through the research and application, we find that using pixel-oriented visualization techniques could make centralized monitoring more effective. More research needs to be carried out to comprehensively monitor the rest of the data shown in Figure 6. As the scale of the SGCC maintenance center continues to expand, the pixel-oriented visualization techniques will play a greater role in system stability.

\section{Acknowledgements}

This work was financially supported by the Science and Technology Project of State Grid (Project No. DZ71-14-046).

\section{REFERENCES}

[1] Ahlberg, C. \& Shneiderman, B. 1994. Visual information seeking: tight coupling of dynamic query filters with star field displays. Proc. Human Factors in Computing Systems CHI 94 Conf. 313-317

[2] Ahlberg, C. et al. 1992. Dynamic Queries for Information Exploration: An Implementation and Evaluation. Proc. ACM CHI Int'l Conf. Human Factors in Computing: 619-626

[3] Anupam, V. et al. 1995. DataSpace: 3-D Visualization of Large Databases. Proc. Int'l Symp. Information Visualization: 82-88

[4] Becker, R.A. et al. 1995. Visualizing network data. IEEE Trans. Visualization and Computer Graphics 1(1): 16-28

[5] Beddow, J. 1990. Shape coding of multidimensional data on a microcomputer display. Proc. Visualization 90: 238-246

[6] Chai, S.J. et al. 2014. Research on Distribution Production Management System in "Centralized Maintenance" Mode. Applied Mechanics and Materials. 631-632(10): 1339-1346 
[7] Herman G.T. \& Levkowitz, H. 1992. Color scales for image data. Computer Graphics and Applications. $72-80$

[8] Inselberg, A. 1985. The plane with parallel coordinates, special issue on computational geometry. The Visual Computer 1: 69-97

[9] Inselberg, A. \& Dimsdale, B. 1990. Parallel coordinates: A tool for visualizing multi-dimensional geometry. Proc. Visualization 90: 361-370

[10] Keim, D.A. 2000. Designing pixel-oriented visualization techniques: theory and applications IEEE Transactions on Visualization and Computer Graphics 6(1): 59-78

[11]Keim, D.A. \& Kriegel, H.P. 1995. Issues in Visualizing Large Databases. Visual Database Systems. The International Federation for Information Processing. 203-214

[12]Pickett, R.M. \& Grinstein, G.G. 1988. Iconographic displays for visualizing multidimensional data. Proc. IEEE Conf. Systems. Man, and Cybernetics. 514-519

[13]Robertson, G.G. et al. 1991.Cone trees: Animated 3d visualizations of hierarchical information. Proc. Human Factors in Computing Systems CHI 91 Conf. 189-194

[14] Schweppe, F. C. \& Handschin, E. J. 1974. Static state estimation in electric power systems. Proc. IEEE. 62: 972 -982

[15] Shuai, L.L. et al. 2013. The discussion of $500 \mathrm{kv}$ centralized monitoring system for large operation and large maintenance mode. Engineering. 5: 127-131

[16]Zhu, D.S. et al. 2009 Centralized remote monitoring system of $500 \mathrm{kV}$ substation. Electric Power Automation Equipment. 29(5): 126-129 